\title{
Thermodynamic optimization of global circulation and climate
}

\author{
Adrian Bejan ${ }^{1, * \dagger}$ and A. Heitor Reis ${ }^{2}$ \\ ${ }^{1}$ Department of Mechanical Engineering and Materials Science, Duke University, Box 90300 , \\ Durham, NC 27708-0300, U.S.A. \\ ${ }^{2}$ Department of Physics, University of Évora, Colegio Luis Verney, Rua Romão Ramalho, 59, \\ 7000-671 Évora, Portugal
}

\begin{abstract}
SUMMARY
The constructal law of generation of flow structure is used to predict the main features of global circulation and climate. The flow structure is the atmospheric and oceanic circulation. This feature is modelled as convection loops, and added to the earth model as a heat engine heated by the Sun and cooled by the background. It is shown that the dissipation of the power produced by the earth engine can be maximized by selecting the proper balance between the hot and cold zones of the Earth, and by optimizing the thermal conductance of the circulation loops. The optimized features agree with the main characteristics of global circulation and climate. The robustness of these predictions, and the place of the constructal law as a selfstanding principle in thermodynamics, are discussed. Copyright (C) 2005 John Wiley \& Sons, Ltd.
\end{abstract}

KEY WORDS: constructal theory; climate; global circulation; thermodynamic optimization

\section{INTRODUCTION}

The purpose of this paper is to establish the connection between two domains: the climate models that have been developed ad hoc in geophysics, and the heat engine models that have been developed ad hoc for wind power calculations in thermodynamics. Both domains are very active, voluminous, and established - to review them is not the objective. The connection is made on the basis of constructal theory (Bejan, 1997, 2000; Bejan et al., 2004), which is the thought that thermodynamic systems far from equilibrium (flow systems) construct flow architectures with purpose: the maximization of global system performance subject to global constraints. The purpose of making the connection is to eliminate the 'ad hoc' from both fields, and to show that a single principle rules all the model optimizations that have been demonstrated so far.

This connection is important because atmospheric and oceanic circulation is the largest flow system on Earth, and because the implications of the theoretical success demonstrated in the two domains are great. In geophysics, the invocation of a principle of maximization of entropy generation rate has been recognized as a 'law of maximum entropy production which follows deductively from the second law of thermodynamics' (Swenson, 1989). If that were the case, the

\footnotetext{
*Correspondence to: Adrian Bejan, Department of Mechanical Engineering and Materials Science, Duke University, Box 90300, Durham, NC 27708-0300, U.S.A.

${ }^{\dagger}$ E-mail: dalford@duke.edu
} 
principle invoked in geophysics is at best a theorem, not a new law. More recent work on constructal theory (Bejan, 2000) suggests that the principle is indeed a self-standing law. The emergence of globally optimized flow architectures in all classes of flow systems (animate, inanimate, engineered) is a universal phenomenon (Bejan, 2000).

Climate models based on the maximization of entropy generation rate began with Malkus' (1954) hypothesis that in Bénard convection the overall heat transfer rate is maximized through the selection of flow pattern. Examples and reviews are available in (Lorenz, 1955; Paltridge, 1975, 1978; Schulman, 1977; North, 1981; Lin, 1982; Lorenz et al., 2001). The connection between this hypothesis and constructal theory was noted in Nelson and Bejan (1998), where the constructal principle delivered all the known characteristics of Bénard flow and heat transfer, in fluids as well as fluid-saturated porous media. In this paper, constructal theory is extended to the problem of atmospheric and oceanic circulation driven by heating from the sun.

The method consists of viewing the Sun-Earth-Universe assembly as an extraterrestrial power plant the power output of which is used for the purpose of forcing the atmosphere and hydrosphere to flow. The power plant models that have been proposed and optimized are listed chronologically in (Bejan, 1988; Gordon and Zarmi, 1989; De Vos, 1992; De Vos and Flater, 1991; De Vos and Van der Wel, 1993). A spaceship, or the Earth, may be viewed as a closed system having two surfaces, a hot surface of area $A_{\mathrm{H}}$ and temperature $T_{\mathrm{H}}$, which is heated by the Sun, and a cold surface $\left(A_{\mathrm{L}}, T_{\mathrm{L}}\right)$ cooled by radiation to the Universe. On a spaceship, the collector $\left(A_{\mathrm{H}}\right)$ and radiator $\left(A_{\mathrm{L}}\right)$ are the object of design (Bejan, 1988). In the modelling of wind generation on Earth, the surfaces $A_{\mathrm{H}}$ and $A_{\mathrm{L}}$ represent the daily illuminated and dark hemispheres (Gordon and Zarmi, 1989; De Vos, 1992; De Vos and Flater, 1991), or the timeaveraged equatorial and polar zones (De Vos, 1992; De Vos and Van der Wel, 1993). In all cases, the total radiation heat transfer surface is fixed,

$$
A_{\mathrm{H}}+A_{\mathrm{L}}=A
$$

Global constraints of this type play a central role in constructal theory. In all the power plant models (Bejan, 1988; Gordon and Zarmi, 1989; De Vos, 1992; De Vos and Flater, 1991; De Vos and Van der Wel, 1993) it was found that the power output can be maximized by selecting two parameters, the area fraction $\left(A_{\mathrm{H}} / A\right)$, and the position of the power plant on the thermodynamic temperature scale, for example, the temperature $T_{\mathrm{H}}$.

Thermodynamic optimization authors (Bejan, 1988; Gordon and Zarmi, 1989; De Vos, 1992; De Vos and Flater, 1991; De Vos and Van der Wel, 1993) are unaware of the use of similar principles in geophysics. At the same time, geophysics authors (Lorenz et al., 2001) continue to be unaware of the thermodynamics literature. To bring these two research worlds together is one of the objectives of this paper. To begin with, none of the power plant models dedicated to the generation of wind on Earth (Bejan, 1988; Gordon and Zarmi, 1989; De Vos, 1992; De Vos and Flater, 1991; De Vos and Van der Wel, 1993) have accounted for natural convection - the mechanism by which the generated power is dissipated into heat by the movement of the atmosphere and the oceans. This mechanism is the new feature that the present paper adds to the power plant models that have been proposed. The mechanism has been recognized before, not only in the modelling of atmospheric dynamics (Lin, 1982; Lorenz et al., 2001), but also in the description of natural convection itself. See, for example, p. 111 in (Bejan, 1984)...the convection loop is equivalent to the cycle executed by the working fluid in a heat engine. In principle, this heat engine cycle should be capable of delivering useful work if we insert 
a propeller in the stream: this is the origin of 'wind power' discussed nowadays in connection with the harnessing of solar work indirectly from the atmospheric heat engine loop. In the absence of work-collecting devices, the heat engine cycle drives its working fluid fast enough so that its entire work output potential is dissipated by friction in the brake at the interface between what moves and what does not move'.

\section{EARTH MODEL WITH NATURAL CONVECTION LOOPS}

The main features of the model are illustrated in Figures 1 and 2. The surface temperature is time independent. It is averaged over the daily and annual cycles, and is represented by two
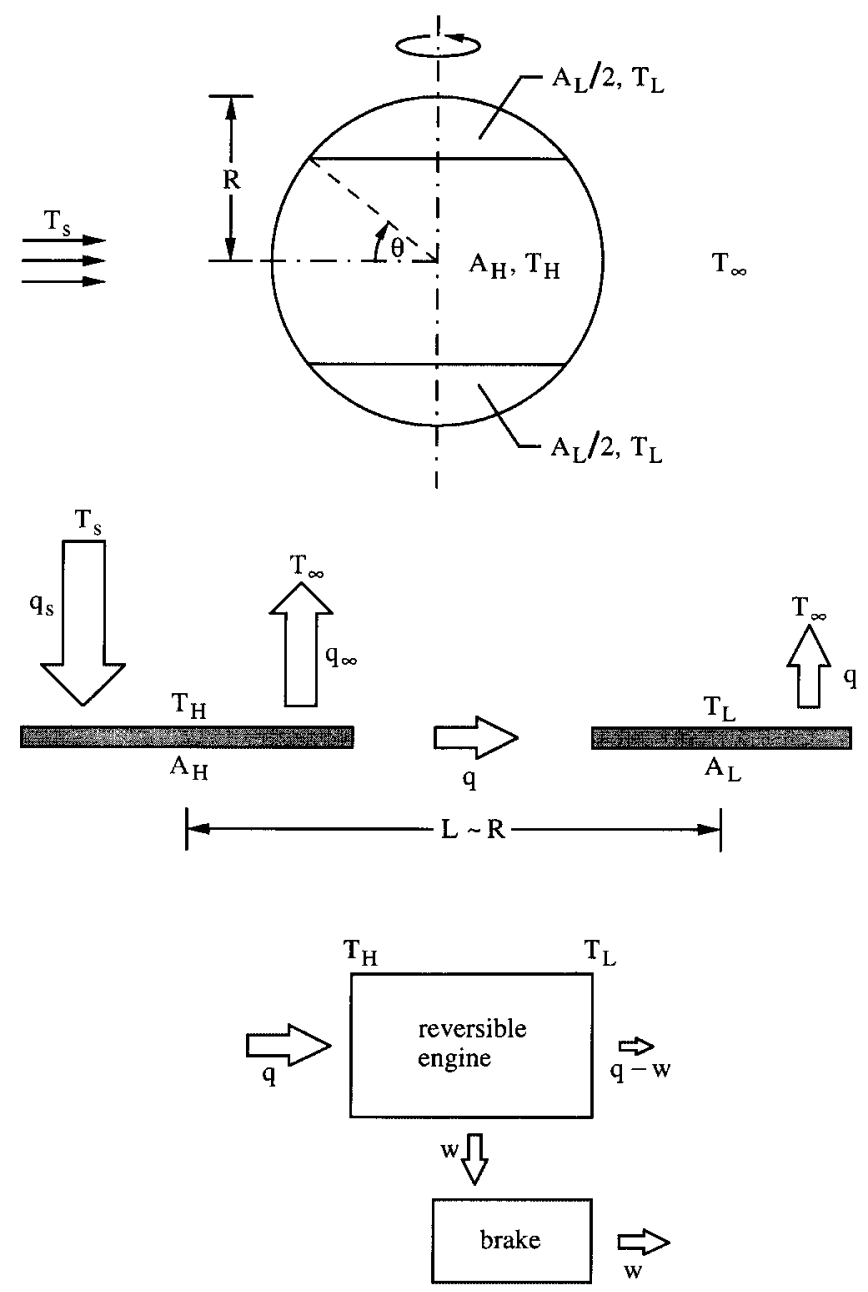

Figure 1. Earth model with equatorial $\left(A_{\mathrm{H}}\right)$ and polar $\left(A_{\mathrm{L}}\right)$ surfaces, and convective heat current between them. 
q

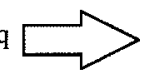

(a)
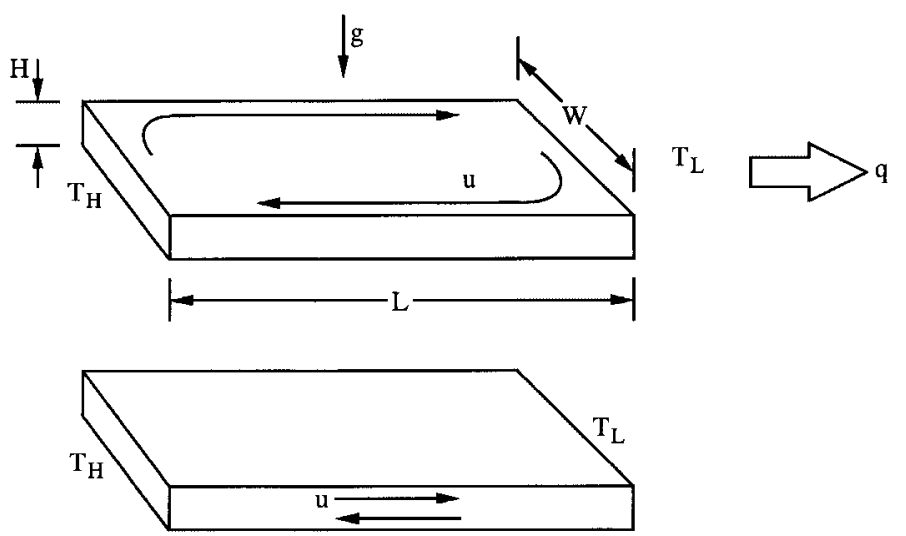

(b)

Figure 2. Natural convection loops in a fluid layer connecting the equatorial and polar surfaces.

temperatures $\left(T_{\mathrm{H}}, T_{\mathrm{L}}\right)$ that correspond to the equatorial and polar zones $\left(A_{\mathrm{H}}, A_{\mathrm{L}}\right)$. This surface model and radiation heat transfer calculations are the same as in De Vos (1992) and De Vos and Van der Wel (1993). The equatorial surface receives the solar heat current

$$
q_{\mathrm{s}}=A_{\mathrm{Hp}}(1-\rho) f \sigma T_{\mathrm{s}}^{4}
$$

where $T_{\mathrm{s}}, \sigma, f$ and $\rho$ are the temperature of the Sun as a black body $(5762 \mathrm{~K})$, the StefanBoltzmann constant $\left(5.67 \times 10^{-8} \mathrm{~W} \mathrm{~m}^{-2} \mathrm{~K}^{-4}\right)$, the Earth-Sun view factor $\left(2.16 \times 10^{-5}\right)$, and the albedo of the Earth (0.3). The area $A_{\mathrm{Hp}}$ is the area $A_{\mathrm{H}}$ projected on a plane perpendicular to the direction Earth-Sun. The ratio $A_{\mathrm{Hp}} / A_{\mathrm{H}}$ decreases from $1 / \pi$ when $A_{\mathrm{H}}$ is a narrow belt along the equator, to $\frac{1}{4}$ when $A_{\mathrm{H}}$ covers the globe almost completely. For simplicity, we adopt the approximation $A_{\mathrm{Hp}} / A_{\mathrm{H}}=\frac{1}{4}$, which is independent of the variable area fraction $x=A_{\mathrm{H}} / A$. This approximation is permissible in view of the order-of-magnitude analysis of the natural convection part of the model.

The $A_{\mathrm{H}}$ surface radiates into space the heat current

$$
q_{\infty}=A_{\mathrm{H}}(1-\gamma) \sigma T_{\mathrm{H}}^{4}
$$

where $\gamma=0.4$ is the Earth's greenhouse factor, or the reflectance in the infrared region (De Vos and Flater, 1991). The difference between $q_{\mathrm{s}}$ and $q_{\infty}$ is convected over the Earth's surface, from $A_{\mathrm{H}}$ to $A_{\mathrm{L}}$

$$
q=q_{\mathrm{s}}-q_{\infty}
$$

and it is finally transmitted by radiation to the cold background,

$$
q=A_{\mathrm{L}}(1-\gamma) \sigma T_{\mathrm{L}}^{4}
$$

Equations (3) and (5) have been simplified by neglecting $T_{\infty}^{4}$ in favour of $T_{\mathrm{L}}^{4}$ and $T_{\mathrm{H}}^{4}$, where $T_{\infty}=3 \mathrm{~K}$ is the temperature of the background, and by considering the Earth emissivity equal to unity. By combining Equations (1)-(5) with the notation $x=A_{\mathrm{H}} / A$, we obtain

$$
T_{\mathrm{H}}^{4}+\left(\frac{1}{x}-1\right) T_{\mathrm{L}}^{4}=B
$$


where $B$ is nearly constant,

$$
B=\frac{f}{4} T_{\mathrm{s}}^{4} \frac{1-\rho}{1-\gamma} \cong 7 \times 10^{9} \mathrm{~K}^{4}
$$

The heat current $q$ is driven from $T_{\mathrm{H}}$ to $T_{\mathrm{L}}$ by the buoyancy effect in the layer of fluid that covers the Earth's surface. In the following derivation of the $q$ formula we neglect factors of order 1, in accordance with the rules of scale analysis (Bejan, 1984). The fluid layer covers an area of flow length $L(\sim R)$ and width $W(\sim R)$, where $R$ is the Earth's radius. The vertical length scale of the fluid layer, $H$, will be defined shortly. The length $L$ bridges the gap between $T_{\mathrm{H}}$ and $T_{\mathrm{L}}$.

At the $T_{\mathrm{H}}$-end of the fluid layer, the hydrostatic pressure at the bottom of the layer is $\rho_{\mathrm{H}} g H$. Similarly, at the $T_{\mathrm{L}}$-end the pressure is $\rho_{\mathrm{L}} g H$. The pressure difference in the $L$ direction is

$$
\Delta P \sim\left(\rho_{\mathrm{L}}-\rho_{\mathrm{H}}\right) g H \sim \rho \beta\left(T_{\mathrm{H}}-T_{\mathrm{L}}\right) g H
$$

where $\rho$ is the mean fluid density, and $\beta$ is the coefficient of volumetric thermal expansion.

The fluid-layer control volume is exposed to the force $\triangle P W H$ in the $L$ direction. This force is opposed by the shear force felt by the moving fluid over the surface $L W$,

$$
\Delta P W H=\tau L W
$$

The average shear stress is

$$
\tau \sim \rho \varepsilon_{\mathrm{M}} \frac{u}{H}
$$

where $\varepsilon_{M}$ is the eddy diffusivity for momentum, and $u$ is the velocity in the $L$ direction. For the order of magnitude of $\varepsilon_{M}$ we use Prandtl's mixing length model (e.g., Bejan, 1984, p. 236), in which we take $H$ to represent the mixing length,

$$
\varepsilon_{\mathrm{M}}=H^{2} \frac{u}{H}=H u
$$

In other words, $H$ is the vertical dimension of the fluid system that mixes (transfers momentum vertically) while moving horizontally. Note that $H$ is not the vertical extent of the fluid layer. By eliminating $\Delta P, u$ and $\varepsilon_{\mathrm{M}}$ between Equations (8)-(11) we obtain the horizontal velocity scale

$$
u \sim\left[\beta g\left(T_{\mathrm{H}}-T_{\mathrm{L}}\right) \frac{H^{2}}{L}\right]^{1 / 2}
$$

The convective heat transfer rate associated with the counterflow between $T_{\mathrm{H}}$ and $T_{\mathrm{L}}$ depends on whether the two branches of the counterflow are in intimate thermal contact. They are not if, for example, the circulation is in the plane $L \times W$, as in the case of $R$-scale oceanic and atmospheric currents that complete loops over large portions of the globe (Figure 2(a)). Another example is when the loop is a vertical plane aligned with the meridian, when the branches of the counterflow are far enough apart and do not exchange heat in a significant way in the vertical direction (Figure 2(b)). In such cases the convective heat current is

$$
q \sim \rho u H W c_{p}\left(T_{\mathrm{H}}-T_{\mathrm{L}}\right)
$$

or, after using Equation (12) and $L \sim W \sim R$,

$$
q \sim \rho c_{p}(g \beta)^{1 / 2} H^{2} R^{1 / 2}\left(T_{\mathrm{H}}-T_{\mathrm{L}}\right)^{3 / 2}
$$


Table I. The effect of the heat transfer model on the results of the double maximization of the power produced and destroyed by global circulation.

\begin{tabular}{lccccr}
\hline$n$ & $w_{\mathrm{mm}}(\mathrm{W})$ & $x_{\mathrm{opt}}$ & $C_{\mathrm{n}, \mathrm{opt}}$ & $T_{\mathrm{L}}(\mathrm{K})$ & $T_{\mathrm{H}}(\mathrm{K})$ \\
\hline 1 & $2.28 \times 10^{8}$ & 0.35 & $1.2 \times 10^{7} \mathrm{~K}^{3}$ & 189.9 & 260.2 \\
$3 / 2$ & $2.28 \times 10^{8}$ & 0.35 & $1.4 \times 10^{6} \mathrm{~K}^{5 / 2}$ & 189.5 & 260.5 \\
2 & $2.28 \times 10^{8}$ & 0.34 & $1.6 \times 10^{5} \mathrm{~K}^{2}$ & 188.1 & 260.0 \\
\hline
\end{tabular}

The convective current is proportional to $\left(T_{\mathrm{H}}-T_{\mathrm{L}}\right)^{3 / 2}$, not to $\left(T_{\mathrm{H}}-T_{\mathrm{L}}\right)$, as it might have been assumed based on a simple invocation of convection as a mechanism (e.g., Equation (15) below). To verify that Equation (14) provides a realistic estimate of the $q$ scale, note that in the geophysics literature (North, 1981) the group

$$
D \sim \frac{q}{R^{2}\left(T_{\mathrm{H}}-T_{\mathrm{L}}\right)}
$$

is known as the convective conductance in the horizontal direction, expressed per unit of horizontal (earth) area. Note that the proportionality between heat current and temperature difference in the $D$ definition is assumed, not deduced (we return to this aspect in Section 4 and Table I). The value of $D$ is known empirically (Lorenz et al., 2001) to be of the order of $0.6 \mathrm{~W} \mathrm{~m}^{-2} \mathrm{~K}^{-1}$. The theoretical estimate (14) anticipates

$$
D \sim \rho c_{p}(g \beta)^{1 / 2} H^{2} R^{-3 / 2}\left(T_{\mathrm{H}}-T_{\mathrm{H}}\right)^{1 / 2}
$$

which for $R \sim 5000 \mathrm{~km},\left(T_{\mathrm{H}}-T_{\mathrm{L}}\right) \sim 40 \mathrm{~K}$, and air properties evaluated at $0{ }^{\circ} \mathrm{C}$ yields

$$
D \sim 0.14 H^{2} \mathrm{~km}^{-2} \mathrm{~W} \mathrm{~m}^{-2} \mathrm{~K}^{-1}
$$

This estimate agrees with the empirical $D$ value when $H$ is of the order of $2 \mathrm{~km}$, which is a realistic transversal length scale for flows in the atmosphere. If we perform the corresponding calculation using the properties of water at $10^{\circ} \mathrm{C}$, then in Equation (17) the coefficient 0.14 is replaced by 70 , and $D$ matches $0.6 \mathrm{~W} \mathrm{~m}^{-2} \mathrm{~K}^{-1}$ when $H$ is of order $100 \mathrm{~m}$ : this length scale is compatible with the thickness of mixing layers in the ocean.

Consider now the alternative where the two branches of the counterflow are in good thermal contact. If the counterflow is a loop in the vertical plane $L \times H$, with the warm branch flowing from $T_{\mathrm{H}}$ to $T_{\mathrm{L}}$ over the top, and the cold branch returning along the bottom (Figure 2(b)), then Equation (12) continues to hold, but Equation (13) is replaced by

$$
q \sim \rho u H W c_{p} \Delta T
$$

where $\Delta T$ is the top-bottom temperature difference between the two branches of the counterflow, $\Delta T<T_{\mathrm{H}}-T_{\mathrm{L}}$. The temperature of the warm branch decreases from $T_{\mathrm{H}}$ at the warm entrance, to $T_{\mathrm{L}}+\Delta T$ at the cold exit. The enthalpy drop in the horizontal direction is caused by the heat transfer by eddy motion in the vertical direction,

$$
u H\left(T_{\mathrm{H}}-T_{\mathrm{L}}-\Delta T\right) \sim \varepsilon_{\mathrm{H}} L \frac{\Delta T}{H}
$$


where $\varepsilon_{\mathrm{H}}$ is the thermal eddy diffusivity. Using again the mixing length model, $\varepsilon_{\mathrm{H}} \sim u H$, we find that Equation (19) reduces to

$$
\frac{\Delta T}{T_{\mathrm{H}}-T_{\mathrm{L}}} \sim \frac{H}{L} \ll 1
$$

such that the convection current (18) becomes

$$
\begin{aligned}
& q \sim \rho c_{p}(g \beta)^{1 / 2} H^{3} R^{-1 / 2}\left(T_{\mathrm{H}}-T_{\mathrm{L}}\right)^{3 / 2} \\
& D \sim \rho c_{p}(g \beta)^{1 / 2} H^{3} R^{-5 / 2}\left(T_{\mathrm{H}}-T_{\mathrm{L}}\right)^{1 / 2}
\end{aligned}
$$

These estimates for $q$ and $D$ are less realistic because they are smaller by a factor of $H / R \ll 1$ than those of Equations (14) and (16).

In conclusion, the convection model with counterflow branches that are not in intimate thermal contact is more realistic. Such a counterflow poses a minimal convective resistance to heat flow in the flow direction. This feature has been encountered before. The same type of counterflow, i.e. the same minimal resistance principle was used earlier in constructal theory in the prediction of Bénard convection (Nelson and Bejan, 1998) and the optimization of cavities in walls with many horizontal partitions (Bejan, 1980) (see also Bejan, 2000).

In the following analysis, we retain Equation (14) because of the test given under Equation (17). Combining Equations (14) and (5), $A_{\mathrm{L}}=(1-x) A$ and $A=4 \pi R^{2}$, we arrive at

$$
C_{3 / 2}\left(T_{\mathrm{H}}-T_{\mathrm{L}}\right)^{3 / 2} \sim(1-x) T_{\mathrm{L}}^{4}
$$

where the theoretical conductance is

$$
C_{3 / 2}=\frac{\rho c_{p}(g \beta)^{1 / 2} H^{2} R^{1 / 2}}{4 \pi R^{2}(1-\gamma) \sigma} \sim 2.1 \times 10^{5} \mathrm{~K}^{5 / 2}
$$

This $C_{3 / 2}$ value corresponds to air at $1 \mathrm{~atm}$ and $0^{\circ} \mathrm{C}$, and the assumption that $H \sim 2 \mathrm{~km}$.

In summary, the radiation-convection model reduces to Equations (6) and (23), which determine the functions $T_{\mathrm{H}}(x)$ and $T_{\mathrm{L}}(x)$. Unlike in earlier thermodynamic optimizations (Gordon and Zarmi, 1989; De Vos, 1992; De Vos and Flater, 1991; De Vos and Van der Wel, 1993), the only degree of freedom in this configuration is the area fraction $x$. Figure 3 shows the effect of $x$ on $T_{\mathrm{H}}$ and $T_{\mathrm{L}}$. In the limit $x=1$, or $A_{\mathrm{H}}=A$, the Earth's surface has a uniform temperature, $289 \mathrm{~K}$. The constants $B$ and $C_{3 / 2}$ are known, although the $C_{3 / 2}$ estimate is not nearly as accurate as $B$. We may treat the convective conductance $C_{3 / 2}$ as a parameter that accounts for the accuracy of the convection model (more on this in Table I).

The fact that the collector-radiator temperature difference $\left(T_{\mathrm{H}}-T_{\mathrm{L}}\right)$ participates in the model as $\left(T_{\mathrm{H}}-T_{\mathrm{L}}\right)^{3 / 2}$ is in agreement with the Monin-Obukhov similarity theory (Pal Arya, 1988), provided that the vertical length scale $H$ is replaced by the Obukhov length,

$$
L_{\mathrm{O}}=\frac{u^{3} \rho c_{p}}{k g \beta q}
$$

where $k \sim 0.24$ is von Karman's constant. The assumption $H \sim L_{\mathrm{O}}$ is reasonable because the Obukhov length is the scale where the atmospheric shear effects are dominant. 


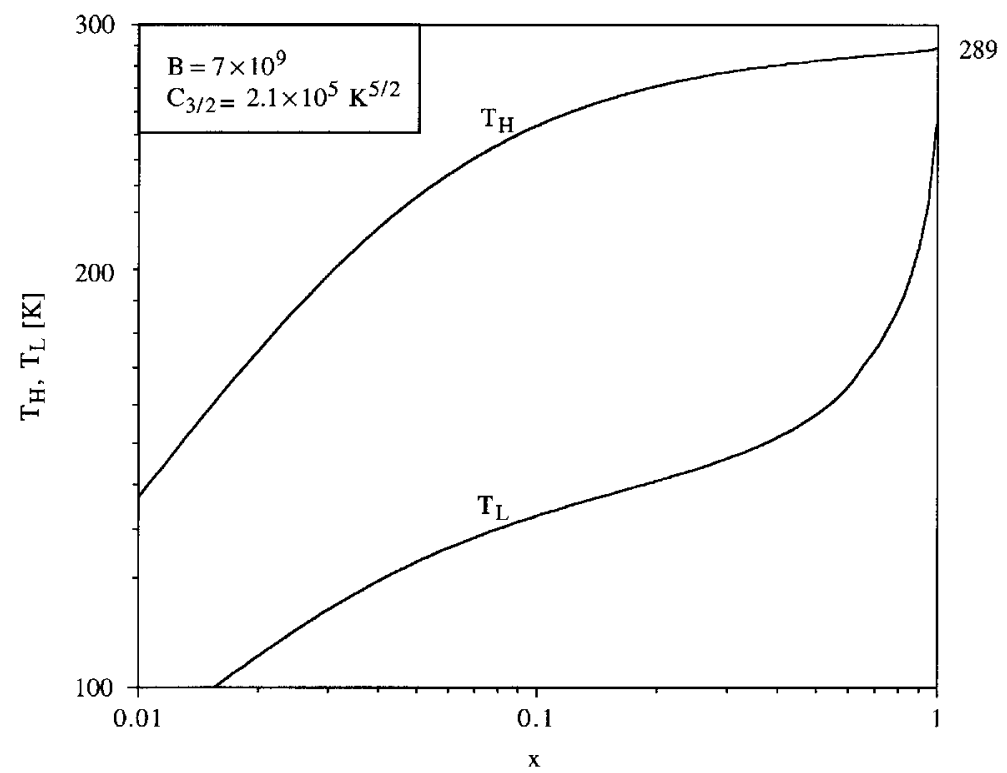

Figure 3. The effect of the area allocation fraction $x$ on the collector and radiator temperatures.

In brief, according to Monin-Obukhov theory, the mean flow and turbulent characteristics of the Earth boundary layer depend on only four independent parameters: (i) the height $z$ above the earth surface, (ii) the friction velocity $u_{*}=(\tau / \rho)^{1 / 2} \mathrm{cf}$. (Pal Arya, 1988, p. 144), where $u_{*}$ is the same as $u$ of Equations (10) and (11), (iii) the surface kinematic heat flux $q^{\prime \prime} /\left(\rho c_{p}\right)$, and (iv) the buoyancy effect $g \beta$, or $g / T$ (cf. Pal Arya, 1988, p. 157). Buckingham's $\pi$ theorem states that the only dimensionless combination of these four variables consists of $z / L_{\mathrm{O}}$ and the $L_{\mathrm{O}}$ group shown in Equation (25). In the analysis presented in this section we arrived at the same conclusion in a different way. The key assumption was made in Equation (9), where the effective vertical length scale $(H)$ was taken to be the same as the thickness of the zone dominated by shear.

\section{MAXIMIZATION OF MECHANICAL POWER DISSIPATION}

The earth surface model with natural convection loops allows us to estimate several quantities that characterize the global performance of atmospheric and oceanic circulation. We pursue this from the constructal point of view, which is that the circulation itself represents a flow geometry that is the result of the maximization of global performance subject to global constraints.

The first quantity is the mechanical power that could be generated by a power plant operating between $T_{\mathrm{H}}$ and $T_{\mathrm{L}}$, and driven by the heat input $q$. The power output $(w)$ is dissipated by friction in fluid flow (a fluid brake system), and added fully to the heat current $\left(q_{\mathrm{L}}\right)$ that the power plant rejects to $T_{\mathrm{L}}$. The power plant and the fluid brake system occupy the space shown between $A_{\mathrm{H}}$ and $A_{\mathrm{L}}$ in the lower part of Figure 1 . Note the continuity of the heat current $q$ through this composite system, $q=w+q_{\mathrm{L}}$. The most power that the composite system could produce is associated with the reversible operation of the power plant. The power output in this 
limit is proportional to

$$
w=q\left(1-\frac{T_{\mathrm{L}}}{T_{\mathrm{H}}}\right)
$$

which is shown as a function of $x$ in Figure 4. The $w$ maximum is due to two competing trends. When $x$ approaches 1, $q$ tends to zero (see Equation (5)), the Earth's surface becomes isothermal $\left(T_{\mathrm{L}} / T_{\mathrm{H}}=1\right)$, and the power output $w$ is zero. In the opposite limit $(x=0), T_{\mathrm{L}}$ tends to zero (see Equation (6)), and $w$ tends to zero because $q$ vanishes. The maximum power $\left(w_{\mathrm{m}}\right)$ occurs when $x_{\mathrm{opt}}=0.35$. This point of optimal thermodynamic operation $\left(x_{\mathrm{opt}}, w_{\mathrm{m}}, T_{\mathrm{H}}, T_{\mathrm{L}}\right)$ is reported in Figures 5-7, where the abscissa parameter is the convective conductance $C_{3 / 2}$, which is allowed to vary.

The maximized power $\left(w_{\mathrm{m}}\right)$ exhibits a maximum with respect to the convective conductance, as shown in Figure 6 . The maximum is located at $C_{3 / 2 \text {,opt }}=1.4 \times 10^{6} \mathrm{~K}^{5 / 2}$ and $w_{\mathrm{mm}}=$ $2.28 \times 10^{8} \mathrm{~W}$. Note that the optimal convective conductance $C_{3 / 2 \text {,opt }}$ exceeds by a factor of only 6 the value anticipated in Equation (24). In view of the approximate character of the simple model (radiation, Earth surface, convection) employed in this analysis (Figures 1 and 2), we conclude that the agreement between $C_{3 / 2 \text {,opt }}$ and Equation (24) is correct in an order of magnitude sense. On the same basis, we speculate that the natural counterflow structure described in Figure 2 is the result of the tendency of the flow system to dissipate maximum power, such that, if suddenly left alone (in isolation), the flow system would reach equilibrium the fastest. This tendency is evident in all flows, and is another way of stating the constructal law (cf. Bejan, 2000, Chapters 5 and 6).

It is also possible to regard the agreement between Equation (24) and $C_{3 / 2 \text {,opt }}$ as a coincidence. The reason for claiming more than a coincidence is the observation that similar coincidences are

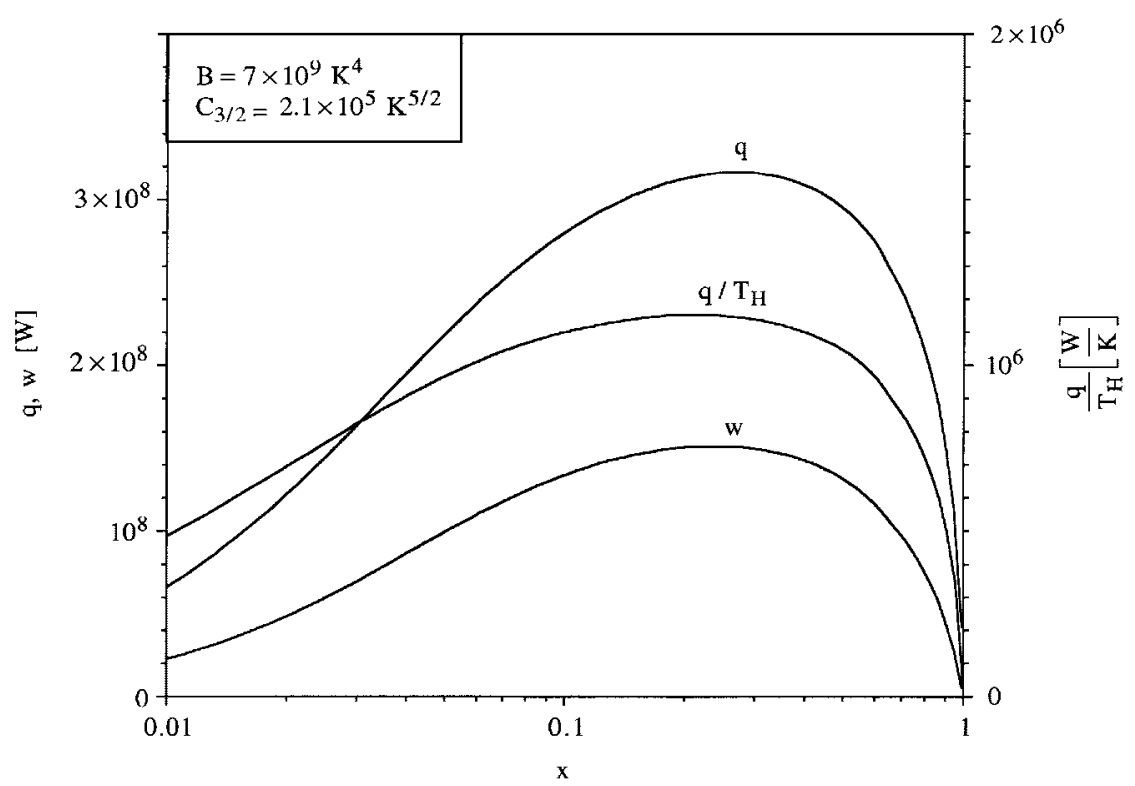

Figure 4. The maximization of power output, convective heat transfer rate, and convection thermal conductance, with respect to the surface allocation ratio. 


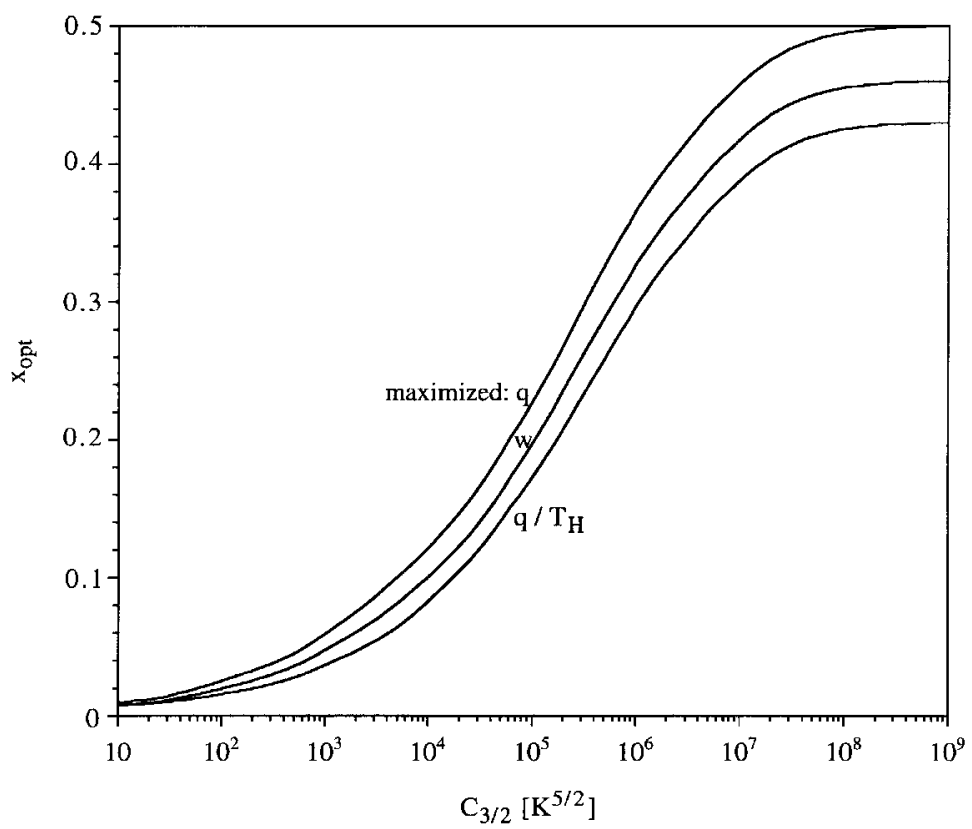

Figure 5. The optimal area allocation ratio resulting from the optimization shown in Figure 4.

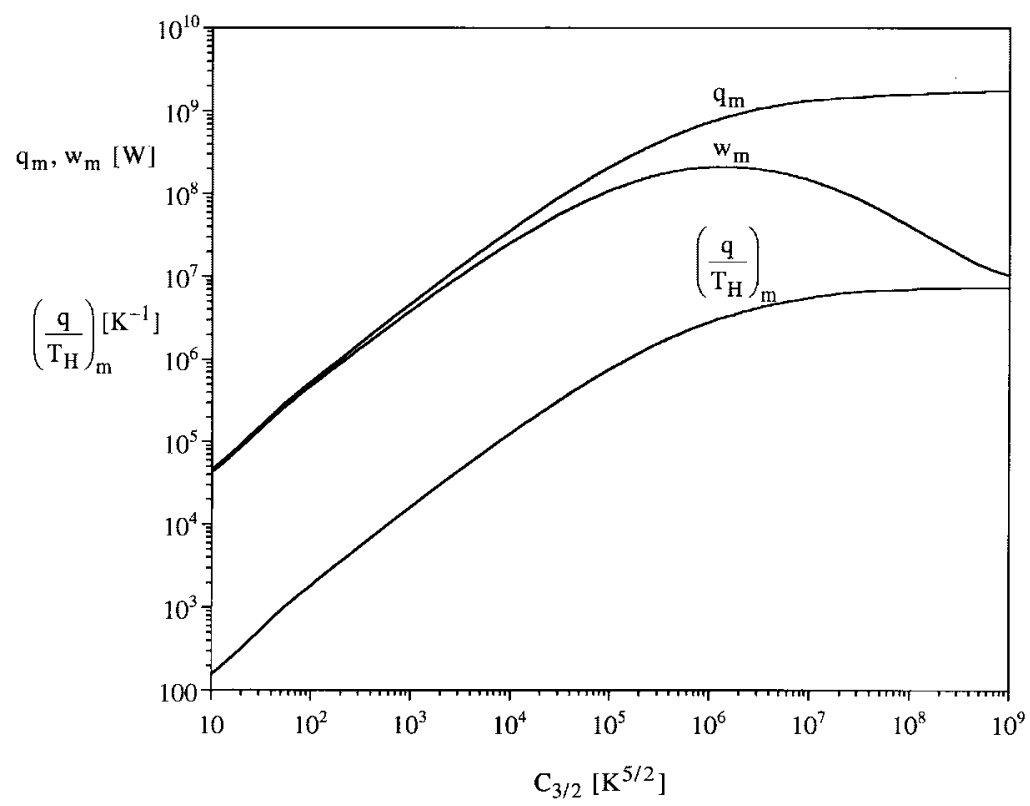

Figure 6. The maximized global performance indicator resulting from the optimizations shown in Figure 4. 


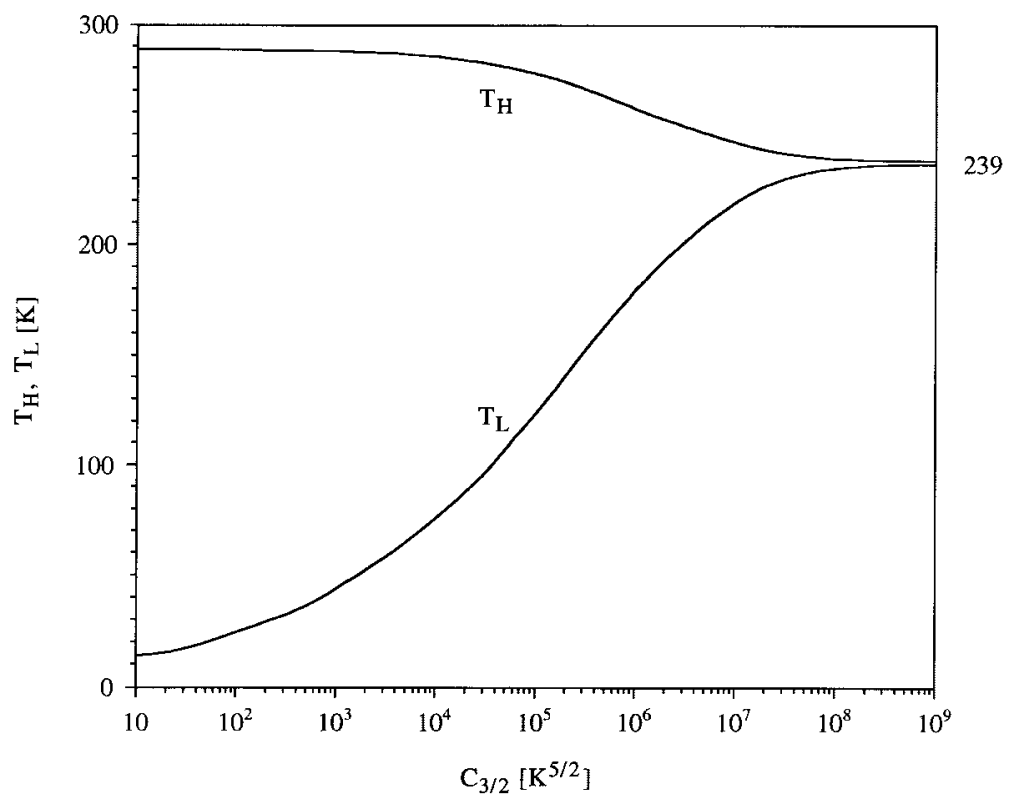

Figure 7. The temperatures of the two surfaces at the optimum summarized in Figures 5 and 6.

occurring every time the global performance maximization principle is invoked. Examples most relevant to the present are the prediction of the flow structure and global Nusselt number for Bénard convection (Nelson and Bejan, 1998) and the laminar-turbulent transition (or turbulent-laminar) in many flow configurations (Bejan, 2000). The present example adds itself to the list, and suggests that global circulation and climate are covered (anticipated) by the constructal law.

\section{THE EFFECT OF EVAPORATION/CONDENSATION OF WATER IN THE ATMOSPHERE}

Better agreement between the present theory and observed properties of atmospheric circulation is obtained if we account for the effect of latent heat in heating and cooling of humid air. In the analysis of Section 2, we used only the sensible heat effect. The ratio between the sensible heat and latent heat effects in the atmosphere is known as the Bowen ratio (Bo). According to Brutsaert (1991), several Bo estimates are available and are of the same order of magnitude, for example: Budyko $(\mathrm{Bo}=5)$, Reichel $(\mathrm{Bo}=3.8)$, and Korzum et al. $(\mathrm{Bo}=5.6)(\mathrm{cf}$. (Brutsaert, 1991) p. 5). The Bo effect can be added to the preceding model by dividing the right side of Equation (14) by Bo.

\section{CONCLUSIONS}

Earlier applications of the constructal principle (Bejan, 2000) recommended it as a self-standing law that is distinct from the second law. There is nothing in classical thermodynamics to require the Carnot power to exhibit maxima with respect to geometric features such as $x$ and $C_{3 / 2}$. That 
the optimized geometric features are consistent with features observed in nature affirms the law status of the principle. It is a thermodynamic principle because it completes the thermodynamic description of flow systems under constraints. Flow and dissipation (irreversibility) must be distributed in special ways in space and time.

Figures 4-6 showed two alternatives to the maximization of power production and dissipation. One is the convective heat current $(q)$ between the two temperature zones. The other is the ratio $q / T_{\mathrm{H}}$, which, in view of the assumption $T_{\infty} \ll T_{\mathrm{H}}$, is essentially the same as the thermal conductance $q /\left(T_{\mathrm{H}}-T_{\infty}\right)$. These two quantities can be maximized with respect to the partitioning of the Earth's surface $(x)$. Figure 5 shows that the optimal area allocation ratio is almost the same as when $w$ is maximized.

The difference is displayed in Figure 6: $q_{\mathrm{m}}$ and $\left(q / T_{\mathrm{H}}\right)_{\mathrm{m}}$ do not exhibit maxima with respect to the convective conductance $C_{3 / 2}$. This makes the maximization and subsequent dissipation of power the more likely objective from the constructal point of view. Why? Because the constructal principle calls for the generation of flow architecture, and architectural features such as $x_{\mathrm{opt}}$ and $C_{3 / 2 \text {,opt }}$ are unambiguously a part of nature.

The robustness exhibited by $x_{\mathrm{opt}}$ with respect to the optimization objective (Figure 5) is intriguing. We may ask whether the natural circulation model has any effect on $x_{\text {opt }}$. For example, according to the simplest heat transfer model of convection, the heat current between the warm and cold regions of the earth would be proportional to the temperature difference $\left(T_{\mathrm{H}}-T_{\mathrm{L}}\right)$, not to $\left(T_{\mathrm{H}}-T_{\mathrm{L}}\right)^{3 / 2}$ as in Equations (14) and (21). This would replace Equation (23) with the more general statement

$$
C_{n}\left(T_{\mathrm{H}}-T_{\mathrm{L}}\right)^{n} \sim(1-x) T_{\mathrm{L}}^{4}
$$

where $n=1$ represents the simplest model, and $n=\frac{3}{2}$ the convection model used in Section 3 . The units of $C_{n}$ are $\mathrm{K}^{4-n}$. Table I shows the results of repeating for $n=1$ and 2 the optimization work described in Section 3. The area allocation ratio and the maximized power are insensitive to the heat transfer model in the $n$ range that can be considered reasonable. The optimized thermal conductance factor $C_{n, \text { opt }}$ depends on the heat transfer model. The important feature of $C_{n}$ is that it can be optimized, i.e. from an infinity of possible $C_{n}$ values, there is one for which the global generation and dissipation of power via organized flow is maximal.

To see why the constructal law of optimization of flow architecture is distinct from the second law, let us review the essence of thermodynamics. Thermodynamic theory was developed in order to account for the functioning and improvement of heat engines. The first law accounts for the conservation of energy, and serves as definition of energy as a concept. The second law accounts for the generation of entropy (irreversibility), on the one-way nature of currents that overcome resistances, and serves as definition of entropy as a concept.

The first law and the second law account for the functioning of a given (observed, assumed) heat engine configuration. The history of flow systems (e.g. heat engines) shows what the first law and the second law are not covering: the case-by-case increase in performance, in time. Every class of flow systems exhibits this behaviour, from river basins to animals and heat engines. Each system in its class represents a flow architecture. New flow systems coexist with old systems, but persist in time if they are better, while older systems gradually disappear. This never-ending parade of flow systems represents the generation of flow architecture - the generation of geometric form as the clash between objective and constraints in flow systems. This is the phenomenon summarized in the constructal law. 
NOMENCLATURE

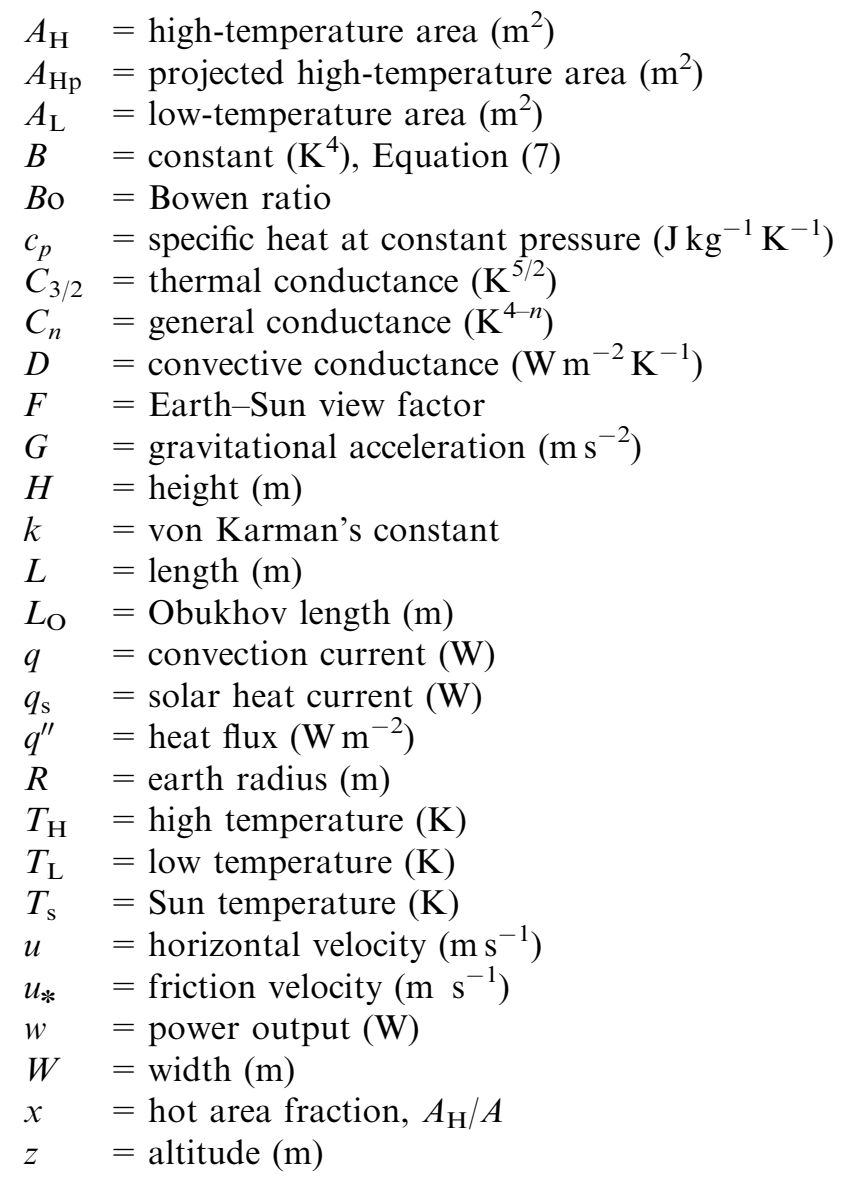

\section{Greek letters}

$\beta=$ coefficient of thermal expansion $\left(\mathrm{K}^{-1}\right)$

$\gamma=$ Earth greenhouse factor

$\Delta P=$ pressure difference $(\mathrm{Pa})$

$\Delta T=$ temperature difference $(\mathrm{K})$

$\varepsilon_{\mathrm{H}} \quad=$ thermal eddy diffusivity $\left(\mathrm{m}^{2} \mathrm{~s}^{-1}\right)$

$\varepsilon_{\mathrm{M}}=$ momentum eddy diffusivity $\left(\mathrm{m}^{2} \mathrm{~s}^{-1}\right)$

$\rho \quad=$ Earth albedo

$\rho_{\mathrm{H}} \quad=$ density of warm air $\left(\mathrm{kg} \mathrm{m}^{-3}\right)$

$\rho_{\mathrm{L}}=$ density of cold air $\left(\mathrm{kg} \mathrm{m}^{-3}\right)$

$\sigma=$ Stefan-Boltzmann constant $\left(\mathrm{W} \mathrm{m}^{-3} \mathrm{~K}^{-4}\right)$

$\tau \quad=$ average shear stress $(\mathrm{Pa})$ 


$$
\begin{aligned}
& \text { Subscripts } \\
& \mathrm{H} \quad=\text { high temperature } \\
& \mathrm{L} \quad=\text { low temperature } \\
& \mathrm{m} \quad=\text { maximized } \\
& \mathrm{mm}=\text { maximized twice } \\
& \mathrm{opt}=\text { optimize }
\end{aligned}
$$

\section{REFERENCES}

Bejan A. 1980. A synthesis of analytical results for natural convection heat transfer across rectangular enclosures. International Journal of Heat and Mass Transfer 23:723-726.

Bejan A. 1984. Convection Heat Transfer. Wiley: New York, 111.

Bejan A. 1988. Advanced Engineering Thermodynamics. Wiley: New York, 520-522.

Bejan A. 2000. Shape and Structure, from Engineering to Nature. Cambridge University Press: Cambridge, UK.

Brutsaert W. 1991. Evaporation into the Atmosphere. Kluwer Academic Publishers: Dordrecht, The Netherlands.

De Vos A. 1992. Endoreversible Thermodynamics of Solar Energy Conversion. Oxford University Press: Oxford, U.K., 53-67.

De Vos A, Flater G. 1991. The maximum efficiency of the conversion of solar energy into wind energy. American Journal of Physics 59:751-754.

De Vos A, Van der Wel P. 1993. The efficiency of the conversion of solar energy into wind energy by means of Hadley cells. Theoretical and Applied Climatology 46:193-202.

Gordon JM, Zarmi Y. 1989. Wind energy as a solar-driven heat engine: a thermodynamic approach. American Journal of Physics 57:995-998.

Lin CA. 1982. An extremal principle for a one-dimensional climate model. Geophysical Research Letters 9:716-718.

Lorenz EN. 1955. Available potential energy and the maintenance of the general circulation. Tellus 7:157-167.

Lorenz RD, Lunine JI, McKay CP, Withers PG. 2001. Titan, Mars and Earth: entropy production by latitudinal heat transport. Geophysical Research Letters 25:415-418.

Malkus WVR. 1954. The heat transport and spectrum of turbulence. Proceedings of the Royal Society Series A 225: 196-212.

Nelson Jr RA, Bejan A. Constructal optimization of internal flow geometry in convection. Journal of Heat Transfer 120: $357-364$.

North GR. Energy balance climate models. Reviews of Geophysics and Space Physics 19:91-121.

Arya PS. 1988. Introduction to Micrometeorology. Academic Press: London.

Paltridge GW. 1975. Global dynamics and climate - a system of minimum entropy exchange. Quarterly Journal of the Royal Meteorological Society 101:475-484.

Paltridge GW. 1978. The steady-state format of global climate. Quarterly Journal of the Royal Meteorological Society 104:927-945.

Schulman LL. 1977. A theoretical study of the efficiency of the general circulation. Journal of the Atmospheric Sciences 34:559-580.

Swenson R. 1989. Emergent attractors and a law of maximum entropy production: foundations to a theory of general evolution. Systems Research 6:187-197. 